\title{
ATFL1 (Anti-TFL1), a novel orthologue induces flowering in the masting alpine snow tussock, Chionochloa pallens (Poaceae)
}

\author{
Samarth ${ }^{* 1}$, Robyn Lee ${ }^{1}$, Dave Kelly ${ }^{1}$, Matthew Turnbull ${ }^{1}$, Richard Macknight ${ }^{2}$, Anthony \\ Poole $^{3}$, and Paula Jameson ${ }^{1}$ \\ ${ }^{1}$ University of Canterbury \\ ${ }^{2}$ University of Otago \\ ${ }^{3}$ The University of Auckland
}

June 25, 2021

\begin{abstract}
Masting, the synchronous highly variable flowering across years by a population of perennial plants, has been shown to be precipitated by many factors including nitrogen levels, drought conditions, spring and summer temperatures. However, the molecular mechanism leading to the initiation of flowering in masting plants in particular years remains largely unknown, despite the potential impact of climate change on masting phenology. We studied genes controlling flowering in Chionochloa pallens, a strongly masting perennial grass. We used a range of in situ and manipulated plants to obtain leaf samples from tillers (shoots) which subsequently remained vegetative or flowered. Here, we show that a novel orthologue of TERMINAL FLOWER 1 (TFL1; normally a repressor of flowering in other species) promotes the induction of flowering in C. pallens (hence Anti-TFL1), a conclusion supported by structural, functional and expression analyses. Global transcriptomic analysis indicated differential expression of CpTPS1, CpGA20ox1, CpREF6 and CpHDA6, emphasising the role of endogenous cues and epigenetic regulation in terms of responsiveness of plants to initiate flowering. Our molecular-based study has provided insights into the cellular mechanism of flowering in masting plants and will supplement ecological and statistical models to predict how masting will respond to global climate change.
\end{abstract}

\section{Introduction}

Mast seeding (or masting) is synchronous highly variable seed production among years by a population of perennial plants (Kelly, 1994; Kelly, Turnbull, Pharis, \& Sarfati, 2008; Schauber et al., 2002). This results in irregular heavy flowering and seeding events, which occur in a range of taxa globally, including in various woody and herbaceous endemic species in the New Zealand flora (Kelly et al., 2008; Schauber et al., 2002; Webb \& Kelly, 1993). A key question is what external and internal factors allow the plants to synchronously trigger heavy reproduction in only some years. A better understanding of those factors, to allow prediction of changes under global climate change (Kelly et al., 2013; McKone, Kelly, \& Lee, 1998; Rees, Kelly, \& Bjornstad, 2002) requires clarification of the underlying genetic mechanisms which control masting (Samarth, Kelly, Turnbull, \& Jameson, 2020).

Although masting imposes costs, such as missed opportunities for reproduction, it is selectively favoured in plants that gain benefits from one or more Economies of Scale (EOS) (Kelly, 1994; Kelly \& Sork, 2002). The two most common EOSs are predator satiation (where seed predators are not able to consume all the seed produced, ensuring higher survival of the offspring) or more efficient wind pollination (Kelly \& Sork, 2002). In order for masting to occur, plants need some synchronising factor, typically a weather cue. Several reports have suggested that a likely cue for masting comes from seasonal changes in summer temperature (Kelly et al., 2013; Schauber et al., 2002) so it has been speculated that increases in global 
temperatures may alter masting behaviour, although the nature of this effect is uncertain (Bogdziewicz, Kelly, Thomas, Lageard, \& Hacket-Pain, 2020; Monks, Monks, \& Tanentzap, 2016; Pearse, LaMontagne, \& Koenig, 2017; Shibata, Masaki, Yagihashi, Shimada, \& Saitoh, 2020). Changes in masting would affect the wider community, potentially impacting on food availability for indigenous seed predators and the rest of the food chain (Touzot et al., 2020).

In recent times, the use of ecological genomics tools has enabled us to determine the molecular nature of ecologically important traits including disease resistance, stress-responsive genes, and agro-economic traits, and their variability among individuals or populations (Richards et al., 2017). Molecular studies, such as those of Miyazaki et al. (2014) and Satake et al. (2019), have shown the potential to improve our understanding of the mechanisms that underpin mast flowering behaviour. Both these papers showed nitrogen levels as proximate drivers of masting in Fagus crenata using resource manipulation and gene expression studies. Similar studies can help show how changes in natural conditions may lead to the evolution of flowering-time genes and associated regulatory mechanisms. However, there is currently little molecular evidence on the mechanisms for temperature-driven mast flowering in plants (Samarth et al., 2021).

Information from model plant species provides useful background to the special case of mast seeding species. Molecular and genetic approaches have revealed that various external cues interact with the developmental processes to regulate the floral transition in perennial plants (Khan, Ai, \& Zhang, 2014; Kobayashi et al., 2013). Genetic pathways controlling flowering time in model crops and temperate grasses, including Arabidopsis (Arabidopsis thaliana), tomato, apple, rice, barley, wheat and Brachypodium distachyon (purple false brome), show a high degree of conservation between dicot and monocot species (Shrestha, Gomez-Ariza, Brambilla, \& Fornara, 2014). Both dicots and monocots share common floral integrator genes including homologues of florigen, the universal flowering hormone. Florigen, or FLOWERING LOCUS T (FT), is a 175 amino acid long protein belonging to the $\mathbf{p}$ hosphatidyl $\mathbf{e}$ thanolamine $\mathbf{b}$ indingp rotein (PEBP) family, an evolutionarily conserved protein family found in all taxa of organisms from bacteria to animals and plants (Karlgren et al., 2011). Phylogenetic analysis of different homologues of the PEBP gene sequences across the plant kingdom has revealed three sub-families. These are MOTHER OF FT AND TFL (MFT), FT and TERMINAL FLOWER 1 (TFL1) (Karlgren et al., 2011). FT and TFL1 protein sequences share $60 \%$ homology with highly conserved amino acid sequences across diverse species. However, these genes act antagonistically to each other: FT promotes flowering whereas TFL1 represses it (Liu, Yang, Wei, \& Wang, 2016).

Most of the core components of the regulatory pathways controlling flowering in temperate grasses are similar to Arabidopsis (Hill \& Li, 2016). However, in contrast to the role of $F L C$ in the vernalisation response in Arabidopsis, the vernalisation response which mediates flowering-time control in temperate monocots is controlled by the VERNALISATION (VRN) loci (Trevaskis, Hemming, Dennis, \& Peacock, 2007). During early growth stages, a floral repressor named VERNALISATION 2 (VRN2), a CCT-domain protein, blocks the floral transition. Exposure to cold temperatures during the winter increases the expression of VERNALISATION 1 (VRN1), a repressor of VRN2 (Yan et al., 2004). During the winter season, VRN1 protein binds to the promoter of VRN2 and blocks its expression (Woods, McKeown, Dong, Preston, \& Amasino, 2016). Post-vernalisation, VRN1 maintains the repressed state of $V R N 2$ through epigenetic changes and releases the VRN2mediated suppression of VERNALISATION 3 (VRN3) (Ream et al. , 2014; Shimada et al. , 2009). VRN3 is an orthologue of FT and Hd3a - the mobile florigen in plants (Shimada et al., 2009). VRN3, expressed in the leaves, then travels to the shoot apical meristem and activates VRN1 to initiate flowering in warm spring conditions (Distelfeld, Li, \& Dubcovsky, 2009).

In the current study, molecular tools were used to investigate the regulation of flowering of the alpine snow tussock, Chionochloa pallens (Poaceae), a temperate non-model grass species. This species is one of the most strongly masting species globally (Kelly et al., 2000), which gives the plant selective benefits through predator satiation (Rees et al., 2002). The possible impacts of global warming on masting in this species have been discussed in the literature (Kelly et al., 2013; McKone et al., 1998; Monks et al., 2016; Rees et al., 2002), but information on the molecular mechanisms inducing flowering is lacking. Understanding the molecular 
regulation of flowering in C. pallenscan provide not only a more accurate prediction of masting years in the face of climate change, but also aid in designing appropriate conservation strategies to save endangered New Zealand fauna (Samarth et al., 2020). From various plants, including some manipulated to induce or prevent flowering, we took leaf samples from tillers (shoots), some of which subsequently flowered and some remained vegetative. We later classified each leaf sample as coming from a plant that subsequently flowered or one from a plant that remained vegetative (Appendix S1). We then used ecological transcriptomics (Samarth et al., 2021, Samarth, Lee, Song, Macknight, \& Jameson, 2019; Todd, Black, \& Gemmell, 2016) to identify the potential homologues of PEBP sequences involved in the onset of flowering. Subsequent structural, functional and expression analysis of the PEBP sequences led to the identification of an orthologous TFL1 gene with a novel function. In addition, the global transcriptomic analysis revealed crucial transcription factors including thermosensory and floral epigenetic genes involved in the initiation of flowering in C. pallens .

\section{Methods}

\section{Study species}

Chionochloa pallens (midribbed snow tussock) is an endemic alpine New Zealand perennial, in the Danthonioideae sub-family of the Poaceae (grasses) (Soreng et al., 2015). Plants grow as tussocks (bunchgrasses) around $0.5-1.5 \mathrm{~m}$ tall and $15-50 \mathrm{~cm}$ in basal diameter. These are very long-lived plants (> 100 years) with discrete individuals. Each individual plant typically comprises hundreds of tillers, each of which in the field is reported to grow for four to five years prior to attaining reproductive maturity (Mark, 1965; Rees et al., 2002). Tillers may then wait some years before switching from vegetative to reproductive, producing a flower stalk (or culm) and typically at least one daughter tiller, before that flowering tiller (but not the rest of the plant) dies. Activation of the inflorescence and floral development in C. pallens occurs a year before flowering (Mark, 1965, and see Appendix S1 in the supplementary information), so we sampled leaves for genetic analysis from marked tillers and stored the samples at $-80{ }^{\circ} \mathrm{C}$ until the fate of that tiller could be determined up to a year later. Informative samples were then selected for RNA analysis. The levels of flowering in C. pallensvary markedly between years (Kelly et al., 2013; Kelly et al., 2000). Previous studies, including transplant experiments to different altitudes, have shown that flowering in Chionochloa is heavier after warm summer temperatures in the year preceding flowering (Mark, 1965; Kelly et al., 2013).

\section{Study site and transplantation experiments}

In low-flowering years, few or no tillers flower in the field, and even in high-flowering years only a minority $(<20 \%)$ of all tillers on each plant flower (Rees et al., 2002). To improve our chances of having both flowering and vegetative samples, we took leaf samples from unmanipulated plants in the field, and from manipulated plants that were transplanted to lower-elevation (warmer) or higher-elevation (colder) sites to make flowering, respectively, more or less likely (Table 1). From 2016 to 2018, unmanipulated C. pallens plants at the main Mt Hutt site at $1070 \mathrm{~m}$ elevation (43032' S, 1710 33' E) were selected with leaf samples taken from three marked tillers on each of 10 marked plants. Only large (full-sized) tillers were selected and tagged for sampling, to avoid smaller tillers that might have been young and reproductively immature, but it was not possible to tell visually which tillers might be going to flower. None of the tagged tillers at the control plot flowered in 2017 and 2018, but some did in 2019 (Table 1).

Several transplantation experiments to higher or lower altitudes were carried out to manipulate flowering in C. pallens. Ten separate plants from the vicinity of the control plot were moved to near sea-level at the University of Canterbury (UC, Christchurch; $43^{\circ} 31^{\prime} \mathrm{S}, 172^{\circ} 35^{\prime} \mathrm{E}, 15 \mathrm{~m}$ above sea level) for the inductive summer period in December 2016 to March 2017 (17Hot transplants) and then transplanted back to the $1070 \mathrm{~m}$ site in March. Leaf samples from tagged tillers (both transplants and control plants) were collected at different times throughout the year (January 2017, March 2017, October 2017 and January 2018; between 11:00 am and 2:00 pm) and were used for the gene expression studies. Leaf samples collected during the inductive summer period (January 2017) were also used for the transcriptomic analysis as described below. Two other sets of 10 plants each were permanently transplanted in 2015 from the control site at $1070 \mathrm{~m}$ to different altitudes: to $1520 \mathrm{~m}$ on Mt Hutt (16Cool) and to UC (16Hot). Leaf samples were collected at 
different seasonal time-points (between 11:00 am and 2:00 pm), including summer (January 2016), autumn (March 2016), spring (October 2016) and autumn (post flowering; March 2017).

In all the experiments, all leaf samples were collected and put directly into vials on dry ice within 20 seconds and stored at $-80^{\circ} \mathrm{C}$ until further analysis. The tagged tillers and the leaf samples from which they were taken were correspondingly labelled and subsequent tiller behaviour (flowering or not) was recorded in the following year. The stored leaf samples could then be identified as being from tillers that subsequently flowered or remained vegetative, and suitable samples were selected for downstream analysis.

\section{Phylogenetic, structural and functional analysis of the PEBP-sequences in C. pallens}

There is no published genome for Chionochloa. All 23 New ZealandChionochloa species have the same chromosome number $(n=7)$ and are regarded as ancient hexaploids $(2 n=6 x=42)$ which have undergone diploidization (Linder \& Barker, 2014; Murray, De Lange, \& Ferguson, 2005). The reference 'C. pallens' transcriptome assembly published in Samarth et al. (2019) was used to identify the potential homologues of the PEBP protein family (Table 2). The identified homologous PEBP sequences were further clustered into their respective sub-families using a phylogenetic approach. The PEBP-phylogenetic tree consisting of 49 protein sequences (Table S1) was reconstructed using the maximum likelihood method with Jukes Cantor substitution matrix and 1000 bootstrap replication using the PhyML package. Homologous PEBP protein sequences were also modelled using a homology modelling approach at the SWISS-PROT server and visualised using Pymol (student version).

For functional analysis, the homologous PEBP-gene sequences $(C p F T 1, C p F T 2, C p F T 3, C p F T 4, C p F T 5$ and $C p T F L 1$ ) were transformed into Arabidopsis accession Landsberg erecta ft-1 mutant plants. The cDNA sequences were cloned into the binary vector (pB2GW7), downstream of the AtSUC2 promoter, and transformed intoAgrobacterium tumefaciens GV3101 by electroporation. Arabidopsisft-1 mutants were transformed using the method of Martinez-Trujillo et al., (2004) as follows: A. tumefaciensharbouring the binary vector were streaked onto LB media plates with selective antibiotics and incubated at $28{ }^{\circ} \mathrm{C}$ for two days. The bacteria were then scraped off the plate and resuspended in $10 \mathrm{~mL}$ of infiltration media (0.5x MS (Duchefa), $0.05 \%$ Silwett L-77 (Lehle Seeds), $5 \%$ sucrose) to an OD600 $=2$ and drops of this solution were pipetted onto unopened flower buds of Arabidopsis. This was repeated three times at $2-3$-day intervals. The resulting seed was sown on seed-raising mix and transformants selected by spraying the young seedlings with Basta herbicide (glufosinate ammonium; Bayer) at $120 \mathrm{mg} / \mathrm{L}$. The number of rosette leaves was counted at the time of flowering. Wild-type Arabidopsis Landsberg erecta plants flower after producing 8-10 leaves whereas $f t-1$ is a late-flowering mutant, flowering after $\sim 20$ leaves (Martinezzapater \& Somerville, 1990).

\section{RNA-extraction}

Three separate biological replicates for RNA sequencing and two separate biological replicates for expression studies, each consisting of three individual leaf samples each from three different plants, were ground under liquid nitrogen using mortar and pestle. Each sample was dissolved in $1 \mathrm{ml}$ of Trizol reagent and heated at $65{ }^{\circ} \mathrm{C}$ for $5 \mathrm{~min}$. The samples were then centrifuged at $13000 \mathrm{~g}$ for $5 \mathrm{~min}$ at $4{ }^{\circ} \mathrm{C}$. RNA was isolated using the Qiagen Plant RNA extraction kit following the manufacturer's instructions. The extracted RNA was further purified using a DNase I digestion kit (Qiagen DNaseI kit) according to the product manual. Extracted RNAs with 260/280 values of 2.0-2.2 and 260/230 values of 1.8-2.0 were selected for downstream analyses. An Illumina TruSeq kit 2.0 was used to prepare cDNA libraries for paired-end sequencing on an Illumina HiSeq 2500 platform. The sequencing was carried out at Macrogen Inc. (South Korea).

\section{Transcriptomic analysis}

Transcriptomic analysis was performed comparing the tillers that flowered in the next season (17Hot) and tillers that remained vegetative (17Control) as described in Samarth et al. (2019). 150 bp generated pairedend reads were assembled into a single reference transcriptome using the Trinity pipeline (Haas et al., 2013). Reads from each of the samples were aligned to the reference assembly to generate a count matrix using Bowtie2.0 (Langmead \& Salzberg, 2012) followed by the differential expression analysis using the DESeq2 
package in R. To accurately identify the significantly differentially expressed genes (adj. p-value $<0.05$ ), stringent parameters including an E-value of $10^{-5}, 70 \%$ query coverage and $50 \%$ protein identity were used for annotation based on orthologous proteins identified in B. distachyon using blastp. Gene ontology and pathway enrichment analysis were performed based on the PANTHER server (Mi et al., 2016). Validation of the RNA-seq results was performed using RT-qPCR with a random selection of four genes from the transcriptomic data (Appendix S2).

\section{Gene expression analysis by RT-qPCR}

Expression analysis was performed as described in Samarth et al. (2019). RNA (1 $\mu \mathrm{g})$ was used to prepare cDNA using a Superscript Reverse Transcriptase III kit (Invitrogen Inc.). The prepared cDNA was diluted 5 -fold with double ionised water and subsequently used in the quantitative PCR (qPCR). The reaction mix contained $7.5 \mu \mathrm{L}$ of Kappa SYBR mix, $4.5 \mu \mathrm{L}$ of water, $1 \mu \mathrm{L}$ each of the forward and reverse primers and 1 $\mu \mathrm{L}$ of cDNA to be used for the gene expression studies. The following PCR program was used to amplify the cDNA during the RT-qPCR reaction: $95{ }^{\circ} \mathrm{C}$ for 10 min hold, followed by 50 cycles of $95{ }^{\circ} \mathrm{C}$ for $10 \mathrm{~s}, 60{ }^{\circ} \mathrm{C}$ for $15 \mathrm{~s}$ and $72{ }^{\circ} \mathrm{C}$ for $20 \mathrm{~s}$. The primer sequences used are listed in Table S2. In addition to CpFT1, CpFT2, CpFT3, CpFT4, CpFT5 and CpTFL1, homologues of other floral-promoting genes known to regulate the expression of FT- like genes (Song et al., 2013), including CpGI ,CpHd1 , CpEhd3 , CpMADS1 , CpTPS1, $C p M A D S 50$ and a temperature regulator of flowering in temperate grasses ( $C p V R N 1$ ) were also included in the expression studies. Despite much effort, the expression of CpFT1 could not be detected in the leaf samples.

\section{Statistical analysis}

Seasonal flowering expression of genes was analysed by two-way ANOVA using the prism software with the following predictors: time-point, site, and their interaction. The detailed statistical analysis is summarised in Table S5. Gene expression data between leaves from plants that subsequently flowered and those from plants that remained vegetative at different altitudes during the inductive summer period were analysed using a two-way student's t-test.

\section{Results}

\section{Flowering in the tillers from which leaf samples were collected}

Control tagged plants at $1070 \mathrm{~m}$ did not flower in 2017 and 2018, but did in 2019, a masting year (Table 1). Since activation of floral development initiates a year before anthesis (Appendix S1), leaf samples from these control plants (18Control; January 2018) were included in the study. Some tillers collected from the plants that had been moved to other altitudes (16Hot, $16 \mathrm{Cool}$, and 17Hot) flowered in the next season (Table 1). Flowering was recorded for each of the tagged tillers and all the leaf samples were then correspondingly identified as being from tillers that subsequently flowered or not (Table 1).

\section{Orthologous PEBP-genes in C. pallens}

Seven distinct homologues of PEBP-like gene family members were identified from the draft transcriptome of $C$. pallens (SRA accession number: GHUI00000000) (Samarth et al., 2019). The GIHRV-domain and LREY/HLHWIV-domain, both characteristic features of PEBP-family proteins, were found to be conserved in the homologous sequences from C. pallens (Fig. 1a).

The general topology of the reconstituted PEBP-protein family tree was consistent with the previous findings of Karlgren et al. (2011) and Liu et al. (2016), indicating that the PEBP phylogenetic tree sub-divides into three major clades. These involve MOTHER OF FT and TFL1 (MFT)-like, FLOWERING LOCUS T (FT)like, and TERMINAL FLOWER1 (TFL1)-like (Fig. 1b). The PEBP-protein family tree is consistent with the species tree. Separate clades for homologous PEBP sequences belonging to monocots and dicots can be seen in the phylogenetic tree. All the C. pallenssequences were found to clade with PEBP protein sequences from species belonging to the Poaceae. Of the seven contigs identified, one belongs to the MFT cluster, 
one belongs to the TFL1 group and five belong to the FT group (Fig. 1b). All sequences grouped with functionally categorised members of their respective families with high bootstrap values.

In $C$. pallens, five of the seven contigs which clustered within the FT group showed the presence of Tyr 85 residue, a critical amino acid for flower promoting activity. These contigs were named CpFT1-CpFT5, respectively (Fig. 1a). The external loop of FT (128-145 amino acid residues), critical for its florigenic activity (Ahn et al., 2006), was conserved in all the C. pallens FT sequences. All the FTs from C. pallens also had conserved characteristic features of an FT protein including His87, Glu109, Arg139, and Gln140 near the ligand-binding pocket. Structural modelling results indicate that Tyr85, His87, and Glu109 can form hydrogen bonds and interact with the Arg139 residue (Fig. S1). However, the Gln140 residue usually found in FT-like sequences has changed to His in CpFT3 and CpFT5, and to Pro in CpFT4 (Fig. 1a). The presence of proline at position 140 in CpFT4 may disturb the ligand-binding wall and may render the protein ineffective.

One of the C. pallens PEBP-like sequences clustering with the TFL1 group has both critical amino acid indicators of a TFL1-like sequence, a His88 and Asp140 (Fig. 1a). Segment B of exon 4 was also seen to be highly variable in $C$. pallens. In genetic complementation of the ft-1 mutant, CpFT1, CpFT2, CpFT3, $C p F T 4, C p F T 5$ and $C p T F L 1$ were all found to accelerate flowering. All the FT/TFL1 -like genes significantly shortened the number of days for the complemented $f t-1$ plants to flower under inductive conditions relative to the mutant (Fig. 1c). This suggests that, in contrast to AtTFL1, which is a known floral repressor (Ahn et al., 2006), the C. pallens TFL1 -like gene promotes the floral transition. Therefore, from here on CpTFL1 is referred to as the ANTI-TFL1 (CpATFL1). It is important to point out that genetic complementation assays using Arabidopsis mutants have been widely used by researchers to test the functionality of genes isolated from highly divergent species including gymnosperms (Karlgren et al., 2011).

\section{Expression of ATFL1 correlates with the induction of flowering in C. pallens}

Leaf samples from the control plants at $1070 \mathrm{~m}$ and transplants that flowered in the next season collected during and after the transplantation period were analysed for the expression of selected flowering-time genes using RT-qPCR (Table 1).

Leaf samples collected from both the 17Hot transplants that flowered in 2018 and control plants (17control) that had remained vegetative showed a seasonal expression pattern $(\mathrm{P}<0.05$; Table S5; Fig. S2). The seasonal expression patterns for CpFT3, CpFT4, CpFT5, CpATFL1, CpVRN1, CpMADS50, CpMADS1 and CpHd1 (Fig. S2) were similar between the control plants at $1070 \mathrm{~m}$, all of which had remained vegetative, and the transplants that flowered in the next season (Table S5). Greater expression of FT-like genes was observed in the spring season (October samples), aligning with studies in model plant species (Nagano et al., 2019). Expression of FT-like genes was similar during the spring season in both leaf sample sets, whether from tillers that flowered in the next season or remained vegetative. However, CpFT1 transcript was not detected in any of the samples due to its expression either being below the limit of detection or because it was not expressed in the leaves of the plants during the time when leaf samples were collected. An increased expression of $C p V R N 1$ (Fig. S2), another floral promoter, was observed during and after the winter season similar to its known seasonal expression pattern in B. distachyon and temperate cereal species (Distelfeld et al., 2009; Woods, Ream, \& Amasino, 2014).

When the expression pattern is compared between the two sets of plants during the inductive summer conditions (January 2017), CpATFL1 had a significantly greater expression in the leaf samples of the 17Hot transplants that flowered in the next season compared to the control plants at $1070 \mathrm{~m}$ that remained vegetative. Along with CpATFL1, other floral promoters including CpTPS1, CpMADS1 ,CpMADS50, and CpVRN1 were also highly expressed in the leaf samples associated with flowering tillers (Fig. 2a, Fig S3).

Similarly using RT-qPCR, the expression of flowering-time genes in the leaf samples from the transplanted plants that had flowered in 2017 (16Hot transplants and 16Cool transplants) was also compared to the control plants at $1070 \mathrm{~m}$ (16Control), none of which flowered. All the gene(s) showed a seasonally variable expression pattern (P < 0.001; Table S5) (Fig. S4). The expression pattern of CpFT2 ,CpFT3 , CpFT4 , CpATFL1, 
CpMADS1,CpEhd3 and CpTPS1 in the leaves of tillers that flowered in the next season at both the sites, UC and $1520 \mathrm{~m}$ (16Hot transplants and 16Cool transplants, respectively) showed a similar expression pattern to that observed in the leaf samples associated with flowering in 17Hot transplants (comparing Fig. S3 and Fig. S4). Even though $C p F T 5$ had a seasonal expression pattern, plants at UC showed greater expression during autumn. All the genes showed a significant differential expression between tillers which subsequently flowered vs tillers that remained vegetative during the inductive summer period $(\mathrm{P}<0.001)$, except for CpFT4 in the $16 \mathrm{Cool}$ transplants (Table S5).

Similar to the 17 Hot transplants, CpATFL1 had a significantly greater expression in the tillers that flowered at both the sites (UC and $1520 \mathrm{~m} ; 16 \mathrm{Hot}$ transplants and 16Cool transplants, respectively) during the inductive summer period (January-2016) (Fig. 2a). Expression analysis of $C p V R N 1$ also correlated with its known seasonal expression pattern with a higher peak in expression post winter (Shimada et al., 2009). The expression of $C p V R N 1$ was greater in the tillers at both the sites (UC and $1520 \mathrm{~m}$ ) that flowered in the next season compared to the plants at the control site that had remained vegetative (Fig. S3). Targeted expression analysis of $C p H d 1, C p G I, C p E h d 3, C p M A D S 50$ and $C p M A D S 1$ showed expression was greater for each of these genes in the leaf samples of the transplanted plants which subsequently flowered compared to the control site plants that remained vegetative (Fig. S3).

Leaf samples associated with tillers that remained vegetative in both the transplants, (16Hot transplant and 16Cool transplants) were found to have significantly lower expression of CpATFL1 during the inductive summer temperature (January 2016) compared to the leaf samples from the same groups of transplants that flowered in the next season (Fig. 2b). In addition to the transplants, leaf samples collected from the control plants during January 2018 (18 Control) that flowered in the masting year 2019 also showed a significantly greater expression of $C p A T F L 1$ compared to the leaf samples from plants that remained vegetative in the year 2019 (Fig. 2b). In summary, expression studies suggest that an elevated expression of CpATFL1 during the inductive summer temperature period is associated with the induction of flowering in C. pallens .

\section{Transcriptomic analysis identifies potential regulators of $C p A T F L 1$}

To study the transcriptional changes associated with the induction of flowering in more depth, differential expression (DE) profiling, using RNA-seq, was performed. Leaf samples (January 2017) from 17Hot transplants located at UC which flowered heavily the next season were compared to the control plants (17Control; January 2017) that remained vegetative in the next season which was a non-masting year.

High-throughput 150 bp paired-end sequencing yielded 32 GB of raw data with 120 million average read counts for each replicate. Reads were assembled into a reference transcriptomic assembly using the Trinity pipeline. The generated de novo assembly yielded 140,826 transcripts, comprised of 383,092 contigs with an average length of $1428.92 \mathrm{bp}$ and an N50 length of $2414 \mathrm{bp}$ (Table S3). A total of 29,566 contigs (adj P < 0.01 ) were significantly differentially expressed (DE) with 14,514 and 15,052 transcripts significantly up and downregulated, respectively (Fig. 3).

Gene Ontology analysis: To gain insights into the function of the genes that were DE, contigs were functionally categorised on the basis of putative biological processes, molecular function, and cellular localisation. Out of 29,566 DE contigs, 15,974 (54.09\%) were annotated against the B. distachyon protein database with an E-value of $10^{-5}$. The DE genes were further categorised based on gene ontology using a hypergeometric test with a significance threshold of 0.05 to identify key correlations between the internal cellular activity and the phenotypic differences.

Upregulated genes in the leaf samples associated with tillers that flowered in the next season were significantly enriched in the cellular components belonging to the cytoplasm, organelle, and intracellular organelle as the top three categories. Proteins encoded by the upregulated genes were further clustered into separate functional categories belonging to protein binding, transferase activity and anion binding. These genes were found to be involved in biological processes enriched in response to stimuli, oxidation-reduction processes and cellular response to stimulus (Fig. 3d). Similarly, gene ontology analysis was also carried out for the proteins encoded by the downregulated transcripts. The downregulated transcripts were significantly enriched in the 
cellular components assigned to the cytoplasm, cell periphery and plasma membrane. These transcripts were then further clustered into distinct molecular functions, with most of them belonging to transferase activity, anion binding, and small molecule binding. Finally, these proteins were assigned to the category of biological processes involved in organo-nitrogen compound metabolic process, protein metabolic process, and biological regulation as the top three classes (Fig. 3d).

2,786 downregulated transcripts when mapped to the KEGG database, were enriched in biosynthesis of secondary metabolites, metabolic pathways and circadian rhythms with a false discovery rate of less than 0.05 (Fig. S5). About 3,788 (12.9\%) transcripts of the upregulated genes were found to be associated with the KEGG pathways. The genes were significantly enriched in metabolic pathways (44.1\%), biosynthesis of secondary metabolites $(26.1 \%)$ and protein processing in the endoplasmic reticulum (4.75\%) as the top three categories.

Differentially expressed orthologues of floral genes in C. pallens: Out of the 29,567 DE contigs, 200 floral protein sequences homologous to Arabidopsis and B. distachyon were significantly differentially expressed in the leaves of the tillers that flowered in the next season compared with tillers that remained vegetative (Table S4). Floral integrator genes, including CpMADS1 and CpATFL1, were highly expressed in the tillers that flowered in the next season, which also aligns with the RT-qPCR analysis described earlier (Fig. 3c; Appendix S2). FRIGIDA (CpFRI), a known floral repressor in Arabidopsis (Choi et al., 2011) and B. distachyon (Higgins, Bailey, \& Laurie, 2010) and other CpFRI -interacting genes were downregulated in the tillers that flowered in the next season (Table S4).

The data derived from the RNA-seq suggests that leaf samples from 17Hot transplants that flowered in the next season also showed an increase in the expression of thermosensory genes including CpPIF4,CpPIF5 and $C p b H L H 80$ relative to plants at the control site. These genes also act as floral promoters in response to high temperatures (Kumar et al., 2012). CpSPL15, a known floral promoter in perennial plants (Hyun et al., 2019), was also upregulated in the 17Hot transplants compared to the control plants. CpVRN2 , a temperature regulated floral repressor (Yan et al., 2004), was downregulated during the inductive summer period in the tillers that subsequently flowered (Fig. 3c).

The RNA-seq data also revealed that two gibberellin catabolism gene family members ( $C p G A 20 x 1$ and $C p G A 20 x 8$ ) were downregulated, while genes involved in gibberellin synthesis, including $C p K S$ (kaurene synthase) and CpGA20ox2, were upregulated in the tillers that subsequently flowered. Gibberellins have been shown to promote flowering in plants either by the activation of $F T$ through SPL-family proteins or by activation of SOC1, independent of FT (Tilmes et al., 2019; Yu et al., 2012).

Several epigenetic editor genes, known to deposit active methylation marks to activate the expression of flowering promoting genes, including CpREF6 , CpMSI1 , CpFLD , and $C p E B S$ (He, 2012), were upregulated in the tillers that flowered in the next season compared to the tillers from the plants at the control site that had remained vegetative (Table S4). Additionally, genes involved in the epigenetic repression of floral repressors, such as CpFLK, CpFY, CpFPA and CpVIL3 (An, Guo, Liu, \& An, 2015), were also upregulated in the tillers that subsequently flowered (Table S4).

\section{Discussion}

Flowering by C. pallens, when growing in natural conditions, is highly variable. Consequently, it is difficult to track the induction of the flowering process using only unmanipulated plants. Transplanting plants to a lower altitude (15 m, University of Canterbury) and warmer temperature conditions induced heavy flowering in $C$. pallens, as previously reported for C. rigida (Mark, 1965), allowing sample collection during the inductive summer period and enabling us to study the molecular regulators of flowering. With recent advances in sequencing technologies, transcriptomic profiling of non-model plant species has been demonstrated to be advantageous and effective when studying ecological phenomena (Miyazaki et al., 2014; Todd et al., 2016). In the present study, the use of ecological transcriptomics enabled the identification of key orthologous flowering-time gene(s) in C. pallens responsible for regulating the floral transition. Transcriptomic profiling of multiple? orthologous floral protein sequences showed that many components of the flowering pathways 
identified in Arabidopsis and B. distachyon were also conserved in $C$. pallens . For example, VRN genes, which are known to regulate the floral transition in response to vernalisation and temperature in temperate grasses (Ream et al., 2014), were also identified successfully using the global transcriptomic approach.

Phylogenetic characterisation followed by expression profiling of the $P E B P$-genes resulted in characterisation of the floral promoting genes. Similar to other species, including Arabidopsis, rice, maize, barley, wheat and $B$. distachyon (Karlgren et al., 2011; Liu et. al., 2016), the identified PEBP-like sequences from C. pallens could be categorised into their potential subfamilies. Five of the sequences were shown to be FT-like sequences. Most of the sequences grouped with sequences which have already been functionally characterised, which aided in predicting function. All of the $C p F T$-like genes showed similar seasonal expression patterns (except for $C p F T 1$ ), with their greatest expression during the spring season. This pattern of expression is similar to the expression of FTs characterised in other plant species (Nagano et al., 2019) but none of them could be correlated with the induction of the floral transition in the tillers that flowered in the next season. This could be because the activation of the floral transition in C. pallensoccurs during summer rather than in spring when the expression of theFT-like genes was at its peak.

\section{CpATFL1 acts as a promoter of flowering in C. pallens}

The TFL1 protein is generally a floral repressor in annual and perennial plants, acting antagonistically to FT (Liu et al., 2016). CpATFL1, clustering with the TFL1 clade in the PEBP -gene family tree, also had the key conserved amino acid signatures of the TFL1 protein, including His88, Asp144, and a variable segment B of exon 4 (Fig. 1a). However, functional analysis, involving genetic complementation of theft-1 mutant of Arabidopsis (Ler ) with CpATFL1, indicated that CpATFL1 acted as a floral promoter: theft-1 mutants complemented with the CpATFL1 gene flowered significantly earlier than the wild-type Arabidopsis. This response prompted a computational biology investigation of CpATFL1. Sequence comparison of CpATFL1 with close relatives of FTin other Poaceae, including $Z C N 7$ and $Z C N 8$ of maize (Lazakis, Coneva, \& Colasanti, 2011), suggested that there is a conserved residue of Gln150 between two different gene sequences. Ho \& Weigel (2014) showed that position 150-152 is conserved in FT -like sequences and is crucial for floral promoting activities (Fig. 4a, b), whereas these positions are much more variable in TFL1 -like sequences. Position 150 in TFL1 -like sequences is generally occupied by either Glu or Asp as observed in ZCN1 and OsRCN4, homologues of TFL1 in maize and rice, respectively (Fig. 4b) (Ho \& Weigel, 2014).

The change in Glu 150 to Gln 150 in CpATFL1 is caused by a single base-pair change from GAA to CAA. Glu is a negatively charged amino acid, while Gln is a positively charged amino acid (Fig. 4c). Ho \& Weigel (2014) showed that altering the surface charge of FT and TFL1 -like sequences can alter their corresponding activities. When analysed for the surface charge potential compared to ZCN8 (an FT homologue in maize) and ZCN1 (a TFL1 homologue in maize), CpATFL1 showed a similar charge distribution to ZCN8. The surface surrounding position 150 was strongly positive in ZCN8 and CpATFL1, whereas the surface around that position in ZCN1 was found to be highly negative (Fig. 4d). This change in the surface charge could be the reason for the flower promoting activity of CpATFL1, in contrast to the usual TFL1 activity.

Our gene expression studies (both RT-qPCR and RNA-seq) also support the hypothesis that CpATFL1 is associated with the induction of flowering in C. pallens (Fig. 2). Elevated expression of CpATFL1 was observed during the inductive summer condition in the leaf samples from the plants that subsequently flowered in all the transplants and plants growing at the control site $(1070 \mathrm{~m})$ in natural conditions. It should be noted that, while it has been accepted that expression of TFL1 -like proteins is restricted to apical meristems (Liu et al., 2016), recent studies have shown that homologous TFL1 -like proteins are expressed in the leaves of several perennial plants, including Arabis alpine (Wang et al., 2011), Rosa chinensis, Fragaria vesca (Iwata et al., 2012), Jatropha curcus (Li et al., 2017), Chrysanthemum morifolium (Gao et al., 2019) and Manihot esculenta (Adeyemo, Hyde, \& Setter, 2019). The specifics of the regulation of TFL1 in perennial plant species is still relatively unknown, except for A. alpina (Wang et al., 2011).

\section{Potential regulators of $C P A T F L 1$ initiate the floral transition in C. pallens}

RNA-seq analysis of the leaves collected during the summer inductive conditions from tillers that subse- 
quently flowered shows upregulation of crucial genes including CpFTIP1 and CpFDP that are involved in the transport of florigen-like molecules from the leaves to the apex to subsequently activate the floral meristem gene(s) through formation of the florigen activation complex (FAC) (Kaneko-Suzuki et al., 2018). Calcium is also required to catalyse the formation of the florigen activation complex (Kawamoto, Sasabe, Endo, Machida, \& Araki, 2015). The expression of $C p C P K 6$, a kinase required for calcium signalling, was also upregulated in the tillers that flowered in the next season. It is important to acknowledge that the formation of the FAC occurs at the shoot apical meristem. Even though the differential expression of these genes was observed in the leaves, it may suggest that CpATFL1 may form the FAC through the activity of CpFTIP1 and CpFDP, catalysed by CpCPK6 at the shoot apical meristem. Further investigation involving a yeast-two hybrid assay or bimolecular fluorescence assay would reveal whether CpATFL1 and CpFTIP1 do indeed interact and could, therefore, transport ATFL1 to the apex.

Vernalisation responses to mediate flowering-time control in temperate monocots are controlled by the $V E R$ NALISATION (VRN) loci (Ream et al., 2014). The VRN2 locus encodes a CCT-domain protein that blocks the floral transition. Exposure to cold temperatures increases the expression of $V R N 1$, a repressor of VRN2 (Yan et al., 2004) and maintains the repressed state of VRN2even after vernalisation. Repression of VRN2 leads to activation of $V R N 3$, a homologue of $F T$ and $H d 3 a$, after plants are exposed to warm spring temperatures (Preston \& Kellogg, 2008; Shimada et al., 2009; Woods, et al., 2016). Transcriptomic analysis (RNA-seq) showed an elevated expression of $C p V R N 1$ in the tillers that flowered during the increase in summer temperature, which is also observed in B. distachyon and temperate cereals during the process of floral transition (Ream et al., 2014; Trevaskis, 2010). On the other hand, expression of CpVRN2 , the repressor of $F T$-like genes (Alexandre \& Hennig, 2007), was downregulated in the same samples. Greater expression of $C p V R N 1$ during the spring season and which remained elevated through the summer may have blocked repressive signals from $C p V R N 2$. This repression was potentially maintained until the next summer resulting in the activated transcription of $C p A T F L 1$ to induce flowering.

Global transcriptomic analysis also revealed an increase in the expression of thermosensory genes, including CpPIF4 and CPPIF5 in the tillers associated with flowering in the next season. PIF genes are known to regulate responses to high temperatures (Choi \& Oh, 2016) and are involved in the activation of the flowering process along with similar bHLH floral promoting proteins such as bHLH76 and bHLH80 (Ito et al., 2012). In addition toPIF -family genes, homologues of bHLH76 and bHLH8Oin C. pallens, were also upregulated in the above samples, which may interact with CpPIF4 to activate ATFL1 . However, transient assays are required to confirm this interaction. The summer temperature cue may also have blocked the expression of several floral repressors, including homologues of AP2-LIKE genes and SVP (Capovilla, Schmid, \& Pose, 2015; Mateos et al., 2015).

As warmer summer temperatures were shown to promote flowering, emphasis was also placed on the role of epigenetic modifiers known to regulate the reproductive transition in plants in response to temperature change. Ambient temperatures have been shown to regulate the expression of floral repressors via epigenetic modification including either through deposition of repressive histone marks (to suppress gene expression) such as H3K27me3 or by removing acetyl groups from the histone tails at the gene loci (Bratzel \& Turck, 2015; He, 2012). In contrast, high temperatures have been shown to lead to the deposition of active histone marks (H3K4me3 or H3K36me3) at the loci of floral promoters (Avramova, 2015). CpREF6 and CpFLD , homologues of REF6 andFLD, which are reported to promote flowering (He, 2012; Lu, Cui, Zhang, Jenuwein, \& Cao, 2011), were upregulated in the leaves of plants that flowered in the next season. CpFLD may then interact with CpHDA6, a histone deacetylase complex, activating the floral promoting genes, a process well established in Arabidopsis ( $\mathrm{Yu}$, Chang, \& Wu, 2016). Homologues of FLK, FVE , and FY, genes known to repress the expression of floral repressors epigenetically (Cho, Yoon, \& An, 2017; He \& Amasino, 2005), were upregulated in the tillers that subsequently flowered. Interestingly, homologues of epigenetic editors, including VIL3 and MSI1, which are known to be involved in the activation of VRN1 and SOC1 , respectively, through deposition of active histone marks (Higgins et al., 2010; Oliver \& Finnegan, 2011; An et al., 2015) were also upregulated in the tillers that flowered in the next season. This may suggest that an external signal such as summer temperatures may lead to certain epigenetic changes enabling the 
transcription of CpATFL1 to promote flowering.

\section{Endogenous signals may also be involved in flower initiation during inductive conditions}

RNA-seq analysis also showed an increased expression of genes involved in gibberellin synthesis in the leaf samples associated with flowering tillers. Previous studies have already shown that gibberellin can initiate and accelerate flowering in Chionochloa sp.in the glasshouse (Martin, Jameson, Mark, Yeung, \& Pharis, 1993) and the field (Turnbull et al., 2012). Gibberellin has also been shown to upregulate the expression of FT and SOC1 independently of the photoperiodic pathway in Arabidopsis and several temperate cereals (Yu et al., 2012). Greater expression of gibberellin synthesis gene(s) may have promoted flowering in C. pallens by escalating the expression of CpATFL1 and CpMADS1 as observed in the RT-qPCR studies. Along with gibberellin, expression of the sugar signalling genes, CpUGT87A2, CpTPS1 and CpHXK1, was found to be significantly greater in the leaves of the tillers that flowered in the next season. Appropriate sugar levels are required as an internal standard for a plant to respond to environmental signals to induce the floral transition (Yang, Xu, Koo, He, \& Poethig, 2013).

These data emphasise that, along with the external signals, $C$. pallens may require accumulation of sufficient internal signals to respond and initiate the floral transition. This may explain why certain tillers in the experimental transplants did not undergo flowering even following the perception of an external cue such as warm summer temperatures. These vegetative tillers may be either too young or maybe still in the process of accumulating enough resources.

\section{Hypothesised molecular model of flowering in C. pallens}

This study showed a strong conservation of the activity of PEBP-like gene family members as floral promoters in C. pallens . The transcriptomic analysis further suggested that C. pallensundergoes flowering through the interaction of external and internal signals which can be integrated at the CpATFL1 locus to initiate flowering.

We suggest that flowering in the masting plant C. pallens is a coordinated two-step process (Fig. 5). As shown in $B$. distachyon, the regulatory loop controlled by the vernalisation loci mediating flowering-time is also conserved in C. pallens. This is the first stage of flowering control in C. pallens, where every year cold winter temperatures may suppress the activity of $C p V R N 2$ by increasing the expression of $C p V R N 1$. The suppression of $C p V R N 2$ remains constant post-vernalisation, thereby leading to the elevated expression of $F T$ -like genes in the spring each year. However, an increase in the FTs does not necessarily correlate with flowering in the next season, as C. pallens flowers intermittently. In the second stage, only plants with sufficient internal cues (higher gibberellin and sucrose levels, and competency to undergo the floral transition) can respond to the warmer summer temperatures. Warmer summer temperatures induce activation of the thermosensory genes (PIF4/5), consequently leading to a greater expression of $C p A T F L 1$. Elevated expression of $C p A T F L 1$ leads on to the floral transition and flowering. This two-stage process almost certainly requires additional epigenetic factors acting on CpATFL1 to control the reproductive transition. Alternatively, plants with insufficient cues may lead to re-activation of $C p V R N 2$, thus allowing plants to remain vegetative for the next season. Further analysis based on CHiP-seq may allow the identification of the specific epigenetic genes and the corresponding methylation changes in the histones to activate the flowering process in response to the environmental cues. This model also supports the known ecological models hypothesized to control flowering through changes in summer temperatures (Kelly et al., 2013, Samarth et al., 2020; Schauber et al., 2002).

Because of the potential impacts of global climate change on masting phenology (Monks et al., 2016), and the potential downstream impacts on introduced and native fauna (Griffiths \& Barron, 2016), it is becoming increasingly important to predict masting years accurately. Such predictions are strongest when they are based on a detailed mechanistic understanding of the underlying control mechanisms. The current study has identified significant molecular regulators of flowering in C. pallens which can be used to explore changes in flowering gene expression under altered climates. This will allow the design of new masting models with greater confidence and to understand how mast flowering may change in the face of climate change. 


\section{Acknowledgements}

The authors acknowledge the Marsden Fund of the Royal Society of New Zealand for providing financial support (Grant UOC1401). The authors acknowledge the IT facilities of the University of Canterbury and the University of Otago for providing the computational facilities and analysis tools.

\section{References}

Adeyemo, O. S., Hyde, P. T., \& Setter, T. L. (2019). Identification of $F T$ family genes that respond to photoperiod, temperature and genotype in relation to flowering in cassava (Manihot esculenta, Crantz). Plant Reproduction, 32 (2), 181-191. doi:10.1007/s00497-018-00354-5

Ahn, J. H., Miller, D., Winter, V. J., Banfield, M. J., Lee, J. H., Yoo, S. Y., . . . Weigel, D. (2006). A divergent external loop confers antagonistic activity on floral regulators FT and TFL1. The EMBO journal, 25 (3), 605-614.

Alexandre, C., \& Hennig, L. (2007). FLC -independent vernalization responses. International Journal of Plant Develpmental Biology, 1 , 202-211.

An, Y., Guo, Y., Liu, C., \& An, H. (2015). BdVIL4 regulates flowering time and branching through repressing miR156 in ambient temperature dependent way inBrachypodium distachyon. Plant Physiology and Biochemistry , 89 , 92-99.

Avramova, Z. (2015). Transcriptional 'memory'of a stress: transient chromatin and memory (epigenetic) marks at stress-response genes. The Plant Journal, 83 (1), 149-159.

Bratzel, F., \& Turck, F. (2015). Molecular memories in the regulation of seasonal flowering: from competence to cessation. Genome Biology, 16 (1), 1.

Bogdziewicz, M., Kelly, D., Thomas, P. A., Lageard, J. G., \& Hacket-Pain, A. (2020). Climate warming disrupts mast seeding and its fitness benefits in European beech. Nature Plants , 6 (2), 88-94.

Capovilla, G., Schmid, M., \& Pose, D. (2015). Control of flowering by ambient temperature. Journal of Experimental Botany, 66 (1), 59-69. doi:10.1093/jxb/eru416

Cho, L. H., Yoon, J., \& An, G. (2017). The control of flowering time by environmental factors. Plant Journal, 90 (4), 708-719. doi:10.1111/tpj.13461

Choi, H., \& Oh, E. (2016). PIF4 integrates multiple environmental and hormonal signals for plant growth regulation in Arabidopsis. Molecules and Cells, 39 (8), 587.

Choi, K., Kim, J., Hwang, H.-J., Kim, S., Park, C., Kim, S. Y., \& Lee, I. (2011). The FRIGIDA complex activates transcription of $F L C$, a strong flowering repressor in Arabidopsis, by recruiting chromatin modification factors. The Plant Cell, 23 (1), 289-303.

Distelfeld, A., Li, C., \& Dubcovsky, J. (2009). Regulation of flowering in temperate cereals. Current opinion in plant biology , 12 (2), 178-184.

Gao, Y. H., Gao, Y. K., Wu, Z. P., Bu, X. L., Fan, M., \& Zhang, Q. X. (2019). Characterization of TERMINAL FLOWER1 homologs CmTFL1cgene from Chrysanthemum morifolium. Plant Molecular Biology, 99 (6), 587-601. doi:10.1007/s11103-019-00838-6

Griffiths, J. W., \& Barron, M. (2016). Spatiotemporal changes in relative rat (Rattus rattus ) abundance following large-scale pest control. New Zealand Journal of Botany, 40 (3), 371-380.

Haas, B. J., Papanicolaou, A., Yassour, M., Grabherr, M., Blood, P. D., Bowden, J., . . . Regev, A. (2013). De novo transcript sequence reconstruction from RNA-seq using the Trinity platform for reference generation and analysis. Nature Protocols, 8 (8), 1494-1512. doi:10.1038/nprot.2013.084 
He, Y., \& Amasino, R. M. (2005). Role of chromatin modification in flowering-time control. Trends in Plant Science, 10 (1), 30-35. doi:10.1016/j.tplants.2004.11.003.

He, Y. H. (2012). Chromatin regulation of flowering. Trends in Plant Science, 17 (9), 556-562. doi:10.1016/j.tplants.2012.05.001

Higgins, J. A., Bailey, P. C., \& Laurie, D. A. (2010). Comparative genomics of flowering time pathways using Brachypodium distachyonas a model for the temperate grasses. PLoS One, 5 (4), e10065.

Hill, C. B., \& Li, C. (2016). Genetic architecture of flowering phenology in cereals and opportunities for crop improvement. Frontiers in plant science, 7 , 1906.

Ho, W. W. H., \& Weigel, D. (2014). Structural Features Determining Flower-Promoting Activity of Arabidopsis FLOWERING LOCUS T. Plant Cell, 26 (2), 552-564. doi:10.1105/tpc.113.115220

Hyun, Y., Vincent, C., Tilmes, V., Bergonzi, S., Kiefer, C., Richter, R., . . . Coupland, G. (2019). A regulatory circuit conferring varied flowering response to cold in annual and perennial plants.Science, 363 (6425), 409. doi:10.1126/science.aau8197

Ito, S., Song, Y. H., Josephson-Day, A. R., Miller, R. J., Breton, G., Olmstead, R. G., \& Imaizumi, T. (2012). FLOWERING BHLH transcriptional activators control expression of the photoperiodic flowering regulator CONSTANS in Arabidopsis. Proceedings of the National Academy of Sciences, 109 (9), 3582-3587.

Iwata, H., Gaston, A., Remay, A., Thouroude, T., Jeauffre, J., Kawamura, K., . . . Foucher, F. (2012). The TFL1 homologue KSN is a regulator of continuous flowering in rose and strawberry. Plant Journal, 69 (1), 116-125. doi:10.1111/j.1365-313X.2011.04776.x

Kaneko-Suzuki, M., Kurihara-Ishikawa, R., Okushita-Terakawa, C., Kojima, C., Nagano-Fujiwara, M., Ohki, I., . . . Taoka, K.-I. (2018). TFL1-like proteins in rice antagonize rice FT-like protein in inflorescence development by competition for complex formation with 14-3-3 and FD.Plant and Cell Physiology, 59 (3), 458-468.

Karlgren, A., Gyllenstrand, N., Kallman, T., Sundstrom, J. F., Moore, D., Lascoux, M., \& Lagercrantz, U. (2011). Evolution of the PEBP gene family in plants: functional diversification in seed plant evolution. Plant Physiology, 156 (4), 1967-1977.

Kawamoto, N., Sasabe, M., Endo, M., Machida, Y., \& Araki, T. (2015). Calcium-dependent protein kinases responsible for the phosphorylation of a bZIP transcription factor FD crucial for the florigen complex formation. Scientific Reports, 5 . doi:UNSP 834110.1038/srep08341

Kelly, D. (1994). The evolutionary ecology of mast seeding. Trends in Ecology $E 3$ Evolution, 9 (12), 465-470.

Kelly, D., Geldenhuis, A., James, A., Penelope Holland, E., Plank, M. J., Brockie, R. E., . . . Maitland, M. J. (2013). Of mast and mean: differential-temperature cue makes mast seeding insensitive to climate change. Ecology Letters, 16 (1), 90-98.

Kelly, D., Harrison, A. L., Lee, W. G., Payton, I. J., Wilson, P. R., \& Schauber, E. M. (2000). Predator satiation and extreme mast seeding in 11 species of Chionochloa (Poaceae). Oikos, 90 (3), 477-488. doi:DOI 10.1034/j.1600-0706.2000.900306.x

Kelly, D., \& Sork, V. L. (2002). Mast seeding in perennial plants: why, how, where? Annual Review of Ecology and Systematics , 427-447.

Kelly, D., Turnbull, M. H., Pharis, R. P., \& Sarfati, M. S. (2008). Mast seeding, predator satiation, and temperature cues inChionochloa (Poaceae). Population ecology, 50 (4), 343-355. doi:10.1007/s10144-0080109-1

Khan, M. R., Ai, X. Y., \& Zhang, J. Z. (2014). Genetic regulation of flowering time in annual and perennial plants. Wiley Interdisciplinary Review RNA, 5 (3), 347-359. doi:10.1002/wrna.1215 
Kobayashi, M. J., Takeuchi, Y., Kenta, T., Kume, T., Diway, B., \& Shimizu, K. K. (2013). Mass flowering of the tropical tree Shorea beccariana was preceded by expression changes in flowering and drought-responsive genes. Molecular Ecology, 22 (18), 4767-4782. doi:10.1111/mec.12344

Kumar, S. V., Lucyshyn, D., Jaeger, K. E., Alos, E., Alvey, E., Harberd, N. P., \& Wigge, P. A. (2012). Transcription factor PIF4 controls the thermosensory activation of flowering. Nature, 484 (7393), 242-245.

Langmead, B., \& Salzberg, S. L. (2012). Fast gapped-read alignment with Bowtie 2. Nature Methods, 9 (4), 357-U354. doi:10.1038/Nmeth.1923

Lazakis, C. M., Coneva, V., \& Colasanti, J. (2011). ZCN8 encodes a potential orthologue of Arabidopsis FT florigen that integrates both endogenous and photoperiod flowering signals in maize. Journal of Experimental Botany, 62 (14), 4833-4842. doi:10.1093/jxb/err129

Li, C. Q., Fu, Q. T., Niu, L. J., Luo, L., Chen, J. H., \& Xu, Z. F. (2017). Three TFL1 homologues regulate floral initiation in the biofuel plant Jatropha curcas. Scientific Reports, 7 . doi:ARTN 4309010.1038/srep43090

Linder, H. P., \& Barker, N. P. (2014). Does polyploidy facilitate long-distance dispersal?. Annals of Botany , $113(7), 1175-1183$.

Liu, Y. Y., Yang, K. Z., Wei, X. X., \& Wang, X. Q. (2016). Revisiting the phosphatidylethanolamine-binding protein (PEBP) gene family reveals cryptic FLOWERING LOCUS T gene homologs in gymnosperms and sheds new light on functional evolution. New Phytologist, 212 (3), 730-744.

Lu, F., Cui, X., Zhang, S., Jenuwein, T., \& Cao, X. (2011). Arabidopsis REF6 is a histone H3 lysine 27 demethylase. Nature Genetics, 43 (7), 715.

Mark, A. (1965). Ecotypic differentiation in Otago populations of narrowleaved snow tussock, Chionochloa rigida. New Zealand Journal of Botany, 3 (4), 277-299.

Martin, M., Jameson, P. E., Mark, A. F., Yeung, E. C., \& Pharis, R. P. (1993). Early panicle development in Chionochloa macra plants induced to flower by 2, 2 dimethyl gibberellin A4 or long days. New Zealand Journal of Botany, 31 (2), 193-201.

Martinezzapater, J. M., \& Somerville, C. R. (1990). Effect of Light Quality and Vernalization on LateFlowering Mutants of Arabidopsis-Thaliana. Plant Physiology, 92 (3), 770-776. doi:DOI 10.1104/pp.92.3.770

Martinez-Trujillo, M., Limones-Briones, V., Cabrera-Ponce, J. L., \& Herrera-Estrella, L. (2004). Improving transformation efficiency of Arabidopsis thaliana by modifying the floral dip method. Plant Molecular Biology Reporter, 22 (1), 63-70.

Mateos, J. L., Madrigal, P., Tsuda, K., Rawat, V., Richter, R., Romera-Branchat, M., . . . Coupland, G. (2015). Combinatorial activities of SHORT VEGETATIVE PHASE and FLOWERING LOCUS C define distinct modes of flowering regulation in Arabidopsis. Genome Biology, 16 . doi:ARTN 3110.1186/s13059015-0597-1

McKone, M. J., Kelly, D., \& Lee, W. G. (1998). Effect of climate change on mast-seeding species: frequency of mass flowering and escape from specialist insect seed predators. Global Change Biology, 4 (6), 591-596.

Mi, H., Huang, X., Muruganujan, A., Tang, H., Mills, C., Kang, D., \& Thomas, P. D. (2016). PANTHER version 11: expanded annotation data from Gene Ontology and Reactome pathways, and data analysis tool enhancements. Nucleic Acids Research, 45 (D1), D183-D189.

Miyazaki, Y., Maruyama, Y., Chiba, Y., Kobayashi, M. J., Joseph, B., Shimizu, K. K., .. \& Satake, A. (2014). Nitrogen as a key regulator of flowering in Fagus crenata : understanding the physiological mechanism of masting by gene expression analysis. Ecology Letters , 17 (10), 1299-1309.

Monks, A., Monks, J. M., \& Tanentzap, A. J. (2016). Resource limitation underlying multiple masting models makes mast seeding sensitive to future climate change. New Phytologist, 210 (2): 419-430. 
Murray, B. G., De Lange, P. J., \& Ferguson, A. R. (2005). Nuclear DNA variation, chromosome numbers and polyploidy in the endemic and indigenous grass flora of New Zealand. Annals of Botany , 96 (7), 1293-1305.

Nagano, A. J., Kawagoe, T., Sugisaka, J., Honjo, M. N., Iwayama, K., \& Kudoh, H. (2019). Annual transcriptome dynamics in natural environments reveals plant seasonal adaptation. Nature Plants, 5 (1), 74-83. doi:10.1038/s41477-018-0338-z

Oliver, S. N., \& Finnegan, E. J. (2011). Epigenetic memory in plants: Polycomb-group regulation of responses to low temperature.Epigenetics: A Reference Manual , 83.

Pearse, I. S., LaMontagne, J. M., \& Koenig, W. D. (2017). Inter-annual variation in seed production has increased over time (1900-2014). Proceedings of the Royal Society B: Biological Sciences , 284 (1868), 20171666.

Preston, J. C., \& Kellogg, E. A. J. P. P. (2008). Discrete developmental roles for temperate cereal grass VERNALIZATION1/FRUITFULL-like genes in flowering competency and the transition to flowering. $146(1), 265-276$.

Ream, T. S., Woods, D. P., Schwartz, C. J., Sanabria, C. P., Mahoy, J. A., Walters, E. M., . . . Amasino, R. M. (2014). Interaction of photoperiod and vernalization determines flowering time ofBrachypodium distachyon . Plant Physiology, 164 (2), 694-709. doi:10.1104/pp.113.232678

Rees, M., Kelly, D., \& Bjornstad, O. N. (2002). Snow tussocks, chaos, and the evolution of mast seeding. American Naturalist, 160 (1), 44-59. doi:Doi 10.1086/340603

Richards, C. L., Alonso, C., Becker, C., Bossdorf, O., Bucher, E., Colome-Tatche, M., .. \& Verhoeven, K. J. (2017). Ecological plant epigenetics: Evidence from model and non-model species, and the way forward. Ecology letters, 20 (12), 1576-1590.

Samarth, Kelly, D., Turnbull, M. H., \& Jameson, P. E. (2020). Molecular control of masting: an introduction to an epigenetic summer memory.Annals of Botany, 125 (6), 851-858. doi:10.1093/aob/mcaa004

Samarth, Lee, R., Kelly, D., Turnbull, M. H., Macknight, R. C., Poole, A. M., \& Jameson, P. E. (2021). Molecular control of the floral transition in the mast seeding plant Celmisia lyallii (Asteraceae). Molecular Ecology , 30 (8), 1846-1863.

Samarth, Lee, R., Song, J., Macknight, R. C., \& Jameson, P. E. (2019). Identification of floweringtime genes in mast flowering plants using de novo transcriptomic analysis. PLoS One, 14 (8), e0216267. doi:10.1371/journal.pone.0216267

Satake, A., Kawatsu, K., Teshima, K., Kabeya, D., \& Han, Q. M. (2019). Field transcriptome revealed a novel relationship between nitrate transport and flowering in Japanese beech. Scientific Reports, 9 . doi:ARTN 432510.1038/s41598-019-39608-1

Schauber, E. M., Kelly, D., Turchin, P., Simon, C., Lee, W. G., Allen, R. B., . . . Brockie, R. (2002). Masting by eighteen New Zealand plant species: the role of temperature as a synchronizing cue. Ecology, 83 (5), 1214-1225.

Soreng, R. J., Peterson, P. M., Romaschenko, K., Davidse, G., Zuloaga, F. O., Judziewicz, E. J., .. \& \& Morrone, O. (2015). A worldwide phylogenetic classification of the Poaceae (Gramineae). Journal of Systematics and Evolution, 53 (2), 117-137.

Shibata, M., Masaki, T., Yagihashi, T., Shimada, T., \& Saitoh, T. (2020). Decadal changes in masting behaviour of oak trees with rising temperature. Journal of Ecology , 108 (3), 1088-1100.

Shimada, S., Ogawa, T., Kitagawa, S., Suzuki, T., Ikari, C., Shitsukawa, N., . . . Murai, K. (2009). A genetic network of flowering-time genes in wheat leaves, in which an APETALA1/FRUITFULL-like gene, VRN1, is upstream of FLOWERING LOCUS T. Plant Journal, 58 (4), 668-681. doi:10.1111/j.1365-313X.2009.03806.x 
Shrestha, R., Gomez-Ariza, J., Brambilla, V., \& Fornara, F. (2014). Molecular control of seasonal flowering in rice, arabidopsis and temperate cereals. Annals of Botany, 114 (7), 1445-1458. doi:10.1093/aob/mcu032

Song, Y.H., Ito, S., \& Imaizumi, T. (2013). Flowering time regulation: photoperiod-and temperature-sensing in leaves. Trends in Plant Science 18 (10): 575-583.

Tilmes, V., Mateos, J. L., Madrid, E., Vincent, C., Severing, E., Carrera, E., .. \& Coupland, G. (2019). Gibberellins act downstream of Arabis PERPETUAL FLOWERING1 to accelerate floral induction during vernalization. Plant physiology, 180 (3), 1549-1563.

Todd, E. V., Black, M. A., \& Gemmell, N. J. (2016). The power and promise of RNA-seq in ecology and evolution. Molecular Ecology, 25 (6), 1224-1241. doi:10.1111/mec.13526

Touzot, L., Schermer, E., Venner, S., Delzon, S., Rousset, C., Baubet, E., . . \& Gamelon, M. (2020). How does increasing mast seeding frequency affect population dynamics of seed consumers? Wild boar as a case study. Ecological Applications , 30 (6), e02134.

Trevaskis, B., Hemming, M. N., Dennis, E. S., \& Peacock, W. J. (2007). The molecular basis of vernalizationinduced flowering in cereals. Trends in plant science, 12 (8), 352-357.

Trevaskis, B. (2010). The central role of the VERNALIZATION1 gene in the vernalization response of cereals. Functional Plant Biology, 37 (6), 479-487.

Turnbull, M. H., Pharis, R. P., Kurepin, L. V., Sarfati, M., Mander, L. N., \& Kelly, D. (2012). Flowering in snow tussock (Chionochloaspp.) is influenced by temperature and hormonal cues. Functional Plant Biology, 39 (1), 38-50.

Wang, R. H., Albani, M. C., Vincent, C., Bergonzi, S., Luan, M., Bai, Y., . . . Coupland, G. (2011). Aa TFL1 confers an age-dependent response to vernalization in perennial Arabis alpina. Plant Cell, 23 (4), 1307-1321. doi:10.1105/tpc.111.083451

Webb, C.J. \& Kelly, D. (1993). The reproductive biology of the New Zealand flora. Trends in Ecology $\&$ Evolution, 8 (12): 442-447.

Woods, D.P., Ream, T., \& Amasino, R. (2014). Memory of the vernalized state in plants including the model grass Brachypodium distachyon. Frontiers in plant science, 5, 99.

Woods, D. P., McKeown, M. A., Dong, Y. X., Preston, J. C., \& Amasino, R. M. (2016). Evolution of VRN2/Ghd7- Like genes in vernalization-mediated repression of grass flowering. Plant Physiology, 170 (4), 2124-2135. doi:10.1104/pp.15.01279

Yan, L., Loukoianov, A., Blechl, A., Tranquilli, G., Ramakrishna, W., SanMiguel, P., . . . Dubcovsky, J. (2004). The wheat VRN2 gene is a flowering repressor down-regulated by vernalization. Science, 303 (5664), 1640-1644.

Yang, L., Xu, M. L., Koo, Y., He, J., \& Poethig, R. S. (2013). Sugar promotes vegetative phase change in Arabidopsis thaliana by repressing the expression of MIR156A and MIR156C .Elife, 2 . doi:ARTN e0026010.7554/eLife.00260

Yu, C.-W., Chang, K.-Y., \& Wu, K. (2016). Genome-wide analysis of gene regulatory networks of the FVE-HDA6-FLD complex in Arabidopsis.Frontiers in plant science, 7 , 555.

Yu, S., Galvao, V. C., Zhang, Y. C., Horrer, D., Zhang, T. Q., Hao, Y. H., . . . Wang, J. W. (2012). Gibberellin regulates the Arabidopsis floral transition through miR156-targeted SQUAMOSA promoter bindinglike transcription factors. Plant Cell, 24 (8), 3320-3332. doi:10.1105/tpc.112.101014

\section{Data accessibility statement}

Accession numbers for the raw sequencing reads submitted to SRA database: 


\section{Author contributions}

Design of the research - Samarth, PEJ, DK and MT

Performance of the research- Samarth and RL

Data analysis- Samarth, RL, RM and DK

Collection, or interpretation- Samarth, RL, RM, AMP, MT, DK and PEJ

Writing the manuscript- Samarth, DK, PEJ, MT and RM

\section{TABLES}

Table 1. Chionochloa pallens plants used for leaf sample collection in this study. All plants were initially growing at $1070 \mathrm{~m}$ on Mt Hutt. The site elevations are in metres above sea level; compared to the $1070 \mathrm{~m}$ site, mean temperatures at $15 \mathrm{~m}$ are about $6 \operatorname{deg} \mathrm{C}$ warmer and at $1520 \mathrm{~m}$ about $2 \operatorname{degC}$ cooler (Kelly et al. 2008). For each row, we took leaf samples from three tagged tillers, from each of 10 tagged plants (20 plants for $16 \mathrm{Cool}$ ). Flowering intensity is the percentage of surviving tillers the following year which flowered (with $\mathrm{N}$ surviving tillers in brackets).

\begin{tabular}{lllll}
\hline Sample set name & Site (with m a.s.l.) & Treatment & Year collected & Fate of sampled tillers the next y \\
\hline 16Control & Hutt $1070 \mathrm{~m}$ & unmanipulated & 2016 & vegetative only \\
17Control & Hutt $1070 \mathrm{~m}$ & unmanipulated & 2017 & vegetative only \\
18Control & Hutt $1070 \mathrm{~m}$ & unmanipulated & 2018 & flowering and vegetative \\
$\mathbf{1 7 H o t}$ & UC $15 \mathrm{~m}$ & moved to $15 \mathrm{~m}$ & 2017 & flowering and vegetative \\
$\mathbf{1 6 H o t}$ & UC $15 \mathrm{~m}$ & moved to $15 \mathrm{~m}$ & 2016 & flowering and vegetative \\
$\mathbf{1 6 C o o l}$ & Hutt $1520 \mathrm{~m}$ & moved to $1520 \mathrm{~m}$ & 2016 & flowering and vegetative \\
\hline
\end{tabular}

Table 2: List of flowering pathway genes selected for expression analysis in C. pallens . The columns represent in sequential order: flowering-time genes identified in C. pallens,corresponding orthologs and sequence identifiers in Arabidopsis and Rice/Purple false broom, percentage identity, E-value and the contig identifier of the corresponding gene identified from the reference transcriptome assembly of $C$. pallens published in Samarth et al., (2019).

\begin{tabular}{llllll}
\hline Gene & Arabidopsis homologue & Arabidopsis locus ID & Rice homologue & Rice protein ID & \% Identi \\
\hline Cp GI & GIGANTEA & $A T 1 G 22770$ & OsGI & CAB56058.1 & 95.24 \\
Cp Ehd3 & & & OsEhd3 & BAI77463.1 & 78.35 \\
Cp MADS50 & & $A T 2 G 45660$ & OsMADS50 & Q9XJ60.1 & 78.39 \\
CpHd1 & CO & $A T 5 G 15840$ & OsHd1 & BAB17628.1 & 73.06 \\
CpTPS1 & TPS1 & $A T 1 G 78580$ & OsTPS1 & BAG89812.1 & 86.09 \\
CpMADS1 & SOC1 & $A T 2 G 45660$ & ZmMADS1 & NP_001105152.1 & 78.8 \\
CpFT2 & $F T$ & $A T 1 G 65480$ & OsHd3a & BAB61030.1 & 85 \\
CpFT3 & $F T$ & $A T 1 G 65480$ & OsHd3a & BAB61030.1 & 62.75 \\
CpFT4 & $F T$ & $A T 1 G 65480$ & OsHd3a & BAB61030.1 & 72.9 \\
CpFT5 & $F T$ & $A T 1 G 65480$ & OsHd3a & BAB61030.1 & 66.06 \\
CpVRN1 & & & XP_010237091.1 & 86.08 \\
CpATFL1 & $T F L 1$ & $A T 5 G 03840$ & ZCNR 1 & ABW96224.1 & 82 \\
\hline
\end{tabular}

\section{Figures}




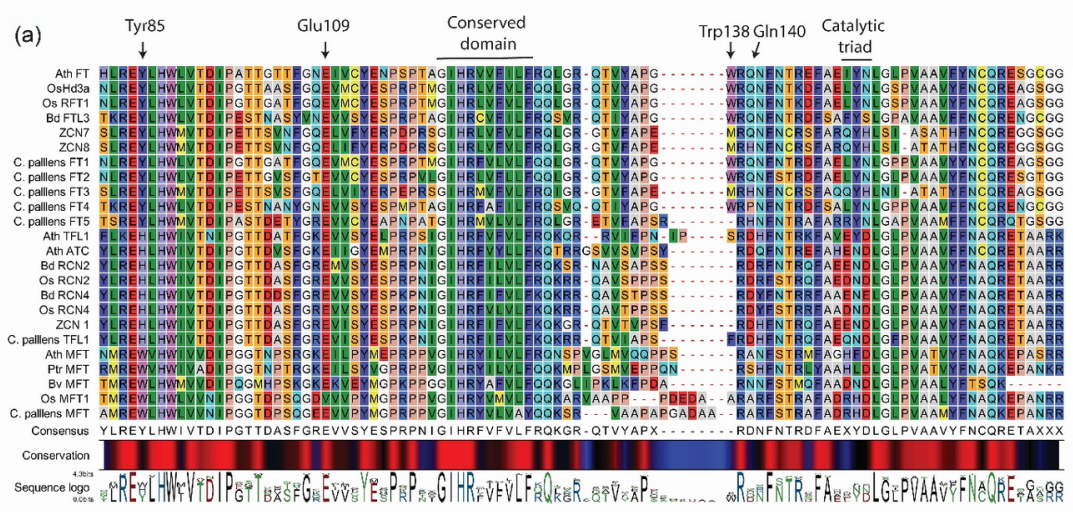

(b)

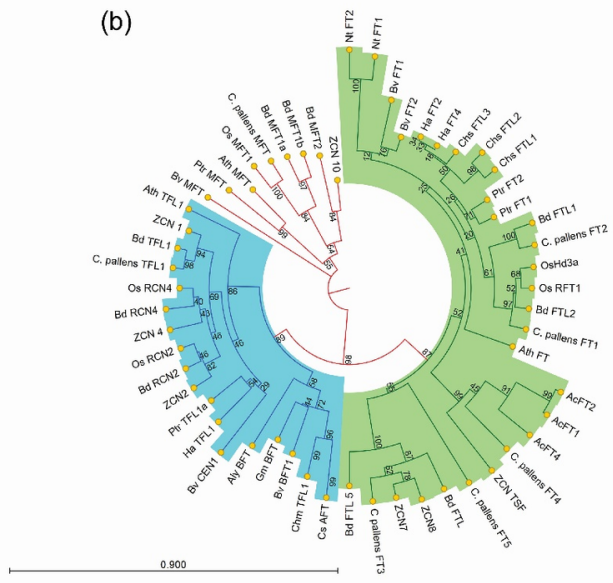

(c)

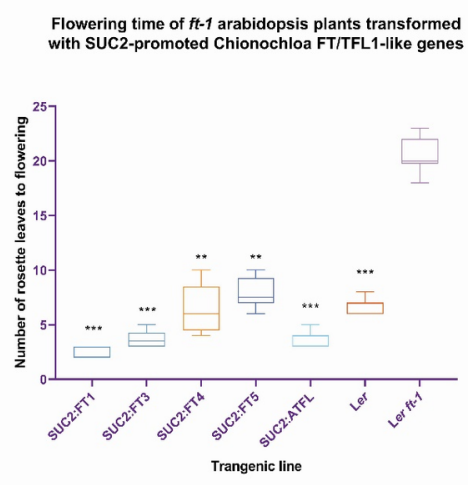

Fig. 1: (a) Multiple sequence alignment of PEBP-like protein sequences. Sequences were aligned using the MUSCLE alignment tool. Sequences are aligned to the 81-172 amino acid long region of FT protein from Arabidopsis. The marked residues/conserved domains are required for the corresponding activity of FT and TFL1-like sequences. The band at the bottom of the alignment represents the percentage of conservation of the amino acids at a position. Red indicates stronger conservation while blue represents variable sequences. (b)Phylogenetic reconstruction of the PEBP-gene family with homologous $C$. pallens sequences - The evolutionary history was inferred by using the Maximum Likelihood method based on the JTT matrix-based model. The bootstrap consensus tree inferred from 1000 replicates is taken to represent the evolutionary history of the taxa analyzed. Aly: Arabidopsis lyrata subsp. lyrata, Ath:Arabidopsis thaliana , Ac: Allium cepa, Bv: Beta vulgaris, Bd: Brachypodium distachyon, Cs:Chrysanthemum seticuspe, Chm: Chrysanthemum X morifolium,Gm: Glycine max, Ha: Helianthus annuus, Nt:Nicotiana tabacum, Osa: Oryza sativa, Ptr:Populus trichocarpa, ZCN: Zea mays and C. pallens. Branches in red represent the MFT clade, blue represents theTFL1 clade and green represents the FT clade. (c) Functional characterization of the $C$. pallens PEBP- like sequences. The number of rosette leaves of the transgenic and the wildtype plants at flowering (mean $\pm \mathrm{SD}, \mathrm{n}=10$ ). (Significance of transgenic and control plants compared to Ler $f t-1$, Student's t-test $\left.{ }^{* * *} \mathrm{P}<0.001,{ }^{* *} \mathrm{P}<0.01\right)$. The $\mathrm{x}$-axis represents Ler as the control plant, Ler ft-1, as the mutant, and the transgenic lines transformed with FT/TFL1 -like sequences from C. pallenscloned into the binary vector pB2GW7 with the AtSUC2 promoter. 

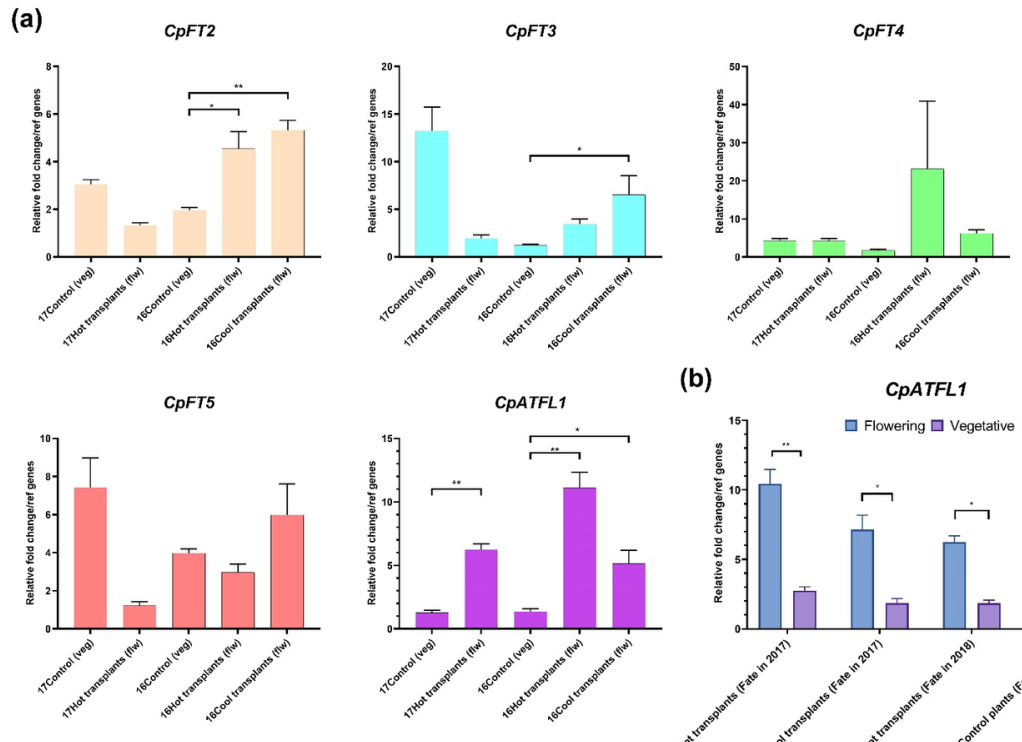

(b)

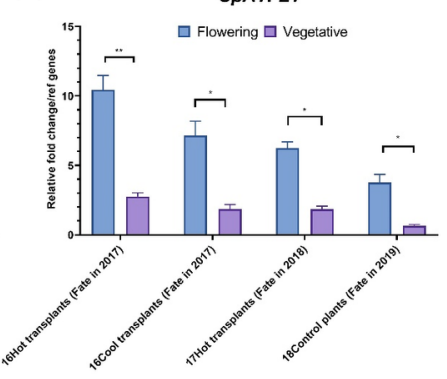

Fig. 2: Gene expression analysis (relative to reference genes) of the orthologous PEBP-sequences in $C$. pallens during the inductive summer period in plants from various sites and fates [either remaining vegetative (veg) or flowering (flw) the next season]. (a) The x-axis indicates the site from where leaf samples were taken: 16Control and 17Control, control plants at $1070 \mathrm{~m}$ in 2016 and 2017; 16Hot and 17Hot, plants translocated from $1070 \mathrm{~m}$ to $15 \mathrm{~m}$ at University of Canterbury sampled in 2016 and 2017; 16Cool, plants transplanted in 2015 from $1070 \mathrm{~m}$ to $1520 \mathrm{~m}$ Mt Hutt sampled in 2016 that flowered in 2017. (b) Expression of CpATFL1 in the leaves of tillers that either flowered or remained vegetative in four separate site/year combinations: from left, University of Canterbury (UC, $15 \mathrm{~m}, 16 \mathrm{Hot}$ ), $1520 \mathrm{~m} \mathrm{Mt} \mathrm{Hutt} \mathrm{(16Cool),} \mathrm{UC} \mathrm{(17Hot),} \mathrm{and} 1070 \mathrm{~m}$ Mt Hutt (18Control). The data are represented by the mean \pm S.D. of two biological replicates each with three technical replicates. The data were calculated using relative fold changes against two internal reference genes, $C p E x P$ and $C p T H P$. (two-way ANOVA, ${ }^{* *} \mathrm{P}<0.01,{ }^{*} \mathrm{P}<0.05$; Table S5). 


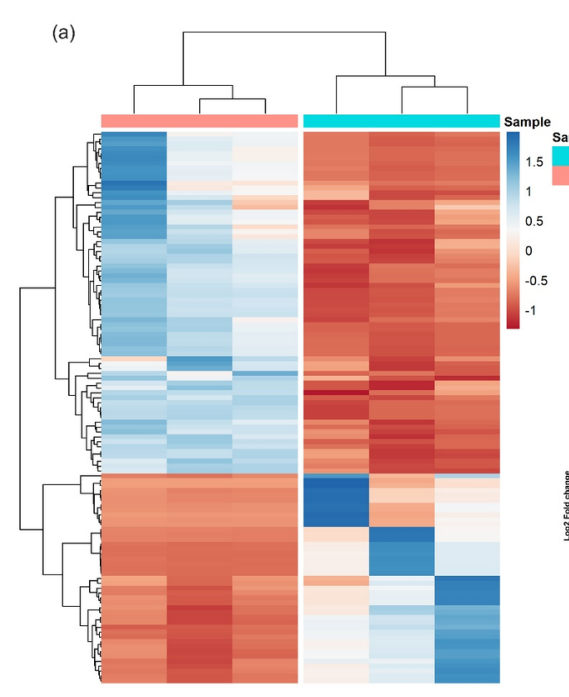

(b)
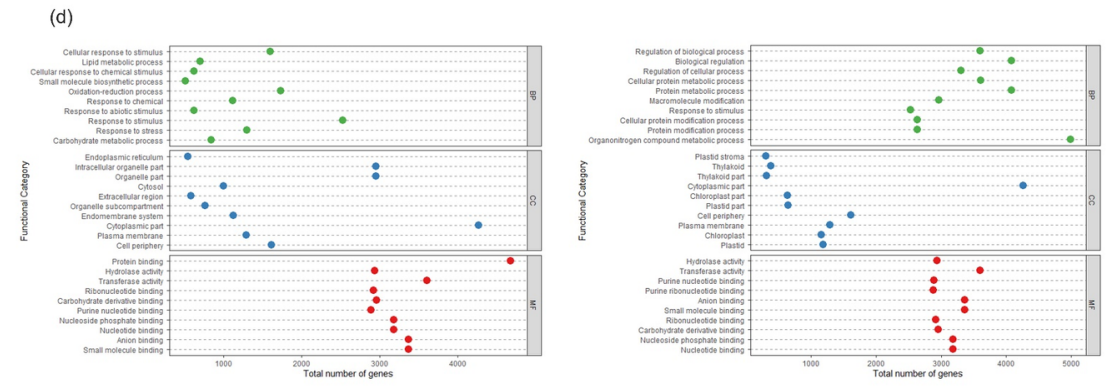

Fig. 3: (a) Heat-map of $\mathbf{5 0}$ most significantly differentially expressed genes in the leaves of $\mathbf{1 7 C o n t r o l}$ and $\mathbf{1 7 H o t}$ transplants $\mathbf{( 1 7 H})$ collected during the inductive summer period (January-2017). The heatmap was constructed using the $\mathrm{R}$ packages DESeq2 and heatmap.2 . Hierarchical clustering is based on the Spearman's coefficient calculated from the log-transformed RNA-seq data. (b) PCA plotof the transcriptomic data from three separate biological replicates, each for 17 Control and 17Hot transplants. (c) Differentially expressed flowering-pathway genes in $\boldsymbol{C}$. pallens. Logarithmic $\left(\log _{2}\right)$ fold change in the orthologous floral genes found to be significantly differentially expressed in the leaves of the 17Hot transplants that flowered in the next season relative to the leaf samples from the control plot plants that remained vegetative. (d)Gene Ontology analysis. GO classification of the upregulated genes (left-hand side) and downregulated genes (right-hand side) in the leaves of the 17Hot transplants that flowered compared to the leaves from the plants at the control plot with a significance level of 0.05 after the Benjamini and Hochberg FDR correlation. (BP: Biological Process, CC: Cellular Component, MF: Molecular Functions) 
(a)

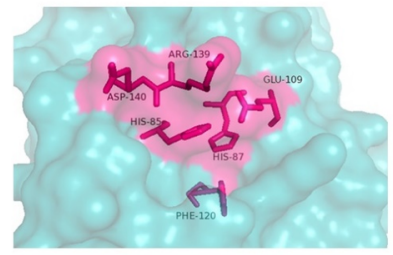

(b)

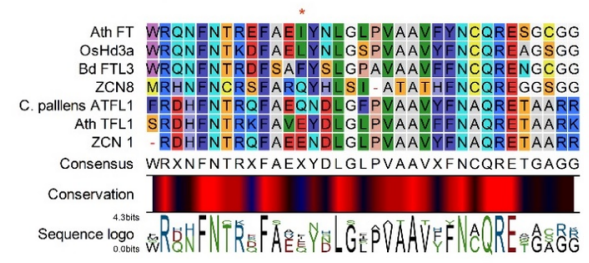

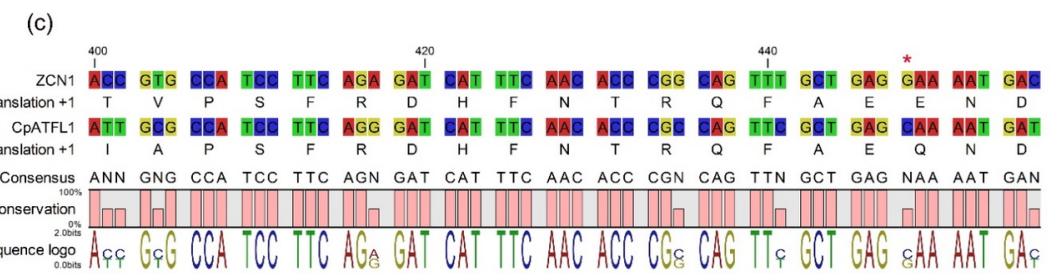

(d)

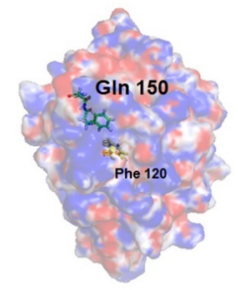

ZCN8

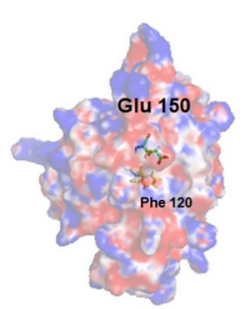

ZCN1

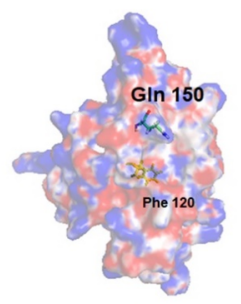

CPATFL1

$-5.000 \quad 5.000$

Fig. 4: (a) Molecular structure of CpATFL1. The protein structure was built based on the ab initio approach from the QUARK server. The highlighted pink region represents the conserved critical amino acids for the activity of TFL1 as a repressor with a reference amino acid, Phe at the position 120, situated towards the bottom region. (b) Multiple sequence alignment of FT and TFL1 homologues in A. thaliana (ATH), Zea mays (ZCN), O. sativa (Os), B. distachyon and C. pallens. Sequences were aligned using the MUSCLE alignment tool. The level of conservation of amino acids is represented by the colour graph at the bottom of the alignment. Red represents stronger conservation while black represents variable amino acid sites. The red asterisk represents the critical amino acid change (Glu-> Gln) in CpATFL1 which may be responsible for its floral-promoting activity. (c) Codon usage between ZCN1, a TFL1 homologue in $Z$. mays, and CpATFL1. * represents the single nucleotide change coding for amino acid at position 150. (d) Electrostatic surface charge for ZCN8, ZCN1 and CpATFL1 . Electrostatic surface potential for each sequence was calculated from the CHARMM server. Protein structures were viewed using the pymol represented by the amino acid at position 150 and a reference amino acid, Phe, at position 120 to orient the structures. 


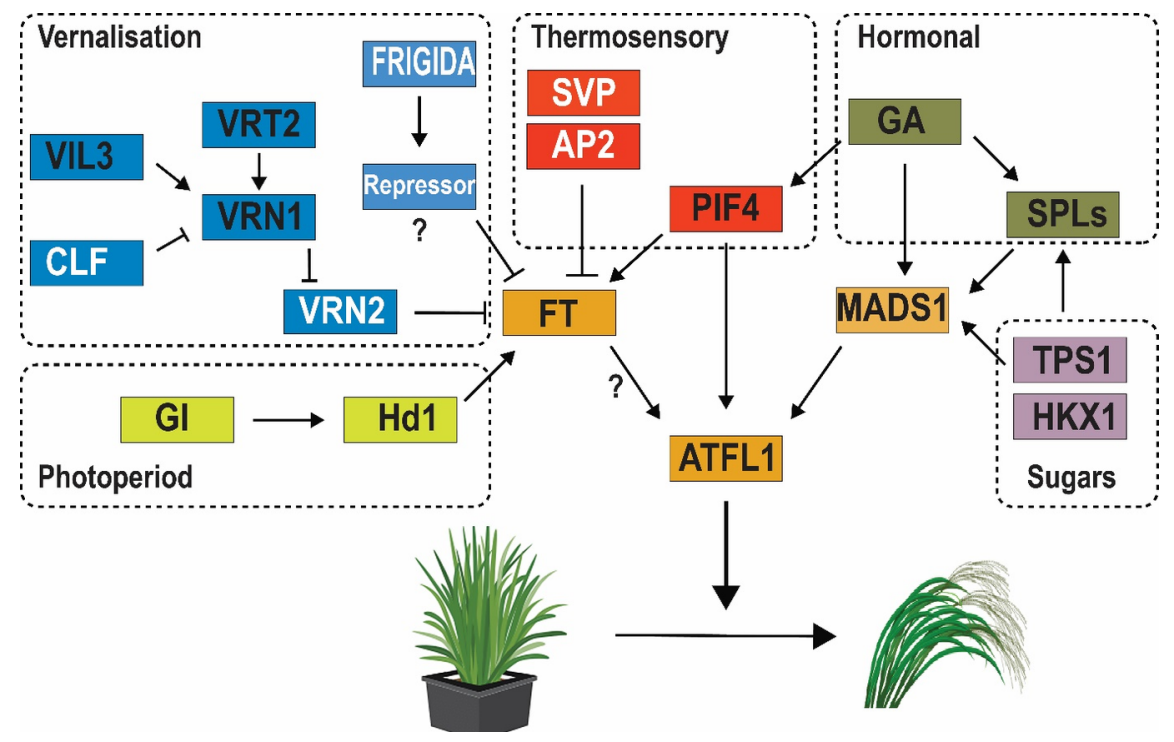

Fig. 5: Hypothesized flowering network in C. pallens. In C. pallens, ATFL1 acts as the key floral integrator gene. During non-inductive conditions, homologues of floral repressors including FRI-like, AP2 and SVP can block the process of floral transition. Photoperiodic (GI, CO) and vernalization (VRN1, VRN2) pathways may interact to activate the expression of $F T$-like genes during spring every year. However, only plants with sufficient internal cues (e.g. sucrose content (TPS1), maturity (SPLs) and gibberellin (GA)) are able to respond to the external signals such as summer temperatures $(P I F 4,5)$ which can then elevate the expression of CpATFL1 to induce flowering in the following season. The orange boxes indicate homologues of the floral integrator genes identified in C. pallens. The solid lines indicate co-expression of genes based on the transcriptomic data and known literature, while the question mark indicates a hypothetical co-regulation between two genes which may not have been discovered yet. Genes represented with black and white ink, respectively represent the up- and down-regulation in the expression of corresponding genes observed in the tillers that flowered in the next season compared to tillers that remained vegetative. 